Check for updates

Cite this: RSC Adv., 2017, 7, 26125

Received 28th March 2017

Accepted 5th May 2017

DOI: $10.1039 / \mathrm{c} 7 \mathrm{ra03570e}$

rsc.li/rsc-advances

\section{Aqueous dispersion of single walled carbon nanotubes stabilized by PEG modified diperylene bisimide and their application as an antibacterial agent $\uparrow$}

\begin{abstract}
Yan Sun (D) ab and Zhibo Li ${ }^{\star b}$
Polyethylene glycol modified diperylene bisimide (PEG-diPBI) was synthesized and applied to disperse single-walled carbon nanotubes (SWCNTs) in water. The aqueous dispersion efficiency of PEG-diPBI on SWCNTs is investigated by UV-vis, Raman spectroscopy, and transmission electron microscopy. It is found that the enhanced dispersing capability of PEG-diPBI can be attributed to the diperylene aromatic core, which can strongly interact with SWCNT via synergistic $\pi-\pi$ and hydrophobic interactions. The dispersing solution properties are closely related to the hydrophilic part of PEG-diPBI. Moreover, the well-dispersed SWCNTs show excellent antibacterial activities.
\end{abstract}

\section{Introduction}

Since the discovery of carbon nanotubes (CNTs) ${ }^{1,2}$ a wide range of their applications ${ }^{3,4}$ have been explored owing to their unique electronic, ${ }^{5}$ mechanical, ${ }^{6}$ and structural properties. ${ }^{7}$ However, pristine CNT tends to aggregate to form bundle structures other than individually dispersed tubes in solvents because of the strong aromatic interactions among CNTs. Thus, improving CNTs' solubility in solvent is of particular importance to expand their practical applications. ${ }^{8,9}$ Generally, covalent modification and non-covalent absorption are two routes for improving CNT solubility. Importantly, non-covalent absorption does not disrupt CNT's electronic structure as well as physical properties, turns out to be an efficient method and economic strategy. On the other hand, perylene bisimide (PBI) is a good macroaromatic dyes. Recently, several amphiphilic PBI derivatives were also demonstrated to be an efficient dispersant for SWCNT. ${ }^{10-13}$ For example, Hirsh and coworkers systematically studied a series of amphiphilic PBI derivatives modified by hydrophilic dendritic polyacids segments to disperse SWCNT in aqueous media, and investigated the dispersing ability dependency on the molecular structure and the side chain. Ernst and coworkers utilized an amphiphilic perylene based surfactant to disperse the carbon nanotubes and to form energy transfer complexes. ${ }^{14}$ Interestingly, the discovery of antibacterial activity has triggered the exploration of applications of CNTs in the field of human health. ${ }^{15-25}$ These findings

${ }^{a}$ College of Chemistry and Chemical Engineering, Yangzhou University, Yangzhou, China, 225002

${ }^{b}$ School of Polymer Science and Engineering, Qingdao University of Science and Technology, Qingdao, China, 266042. E-mail: zbli@qust.edu.cn

$\dagger$ Electronic supplementary information (ESI) available. See DOI: 10.1039/c7ra03570e implied that an improved contact between CNTs and bacteria may lead to a superior antibacterial activity. Kang et al. showed that partially debundled CNTs have higher bacterial toxicity than CNTs bundles. The advent of CNTs in industrial applications raised concerns about the potential impacts of CNTs. Due to the difficulty in debundling SWCNTs and characterizing their aggregation state, to the best of our knowledge, previous studies have utilized either suspended or deposited SWCNT aggregates in the antibacterial activity evaluation other than individually dispersed SWCNTs. Therefore, design and synthesis of new amphiphilic molecules, which can efficiently disperse SWCNTs, are still greatly desirable. We previously reported the PBI derivatives modified SWCNTs. ${ }^{26}$ Novel triply linked diperylene bisimide (diPBI) shows exceptional properties due to extended but distorted $\pi$-system. Here, we use diPBI derivative ${ }^{27}$ as core and PEG as side chains, then investigate their interactions with SWCNT and characterize the corresponding dispersing efficiency in water. We investigated their interactions with SWCNTs to control and manipulate the dispersion efficiency. Furthermore, we observed higher bacterial toxicity when the nanotubes are debundled and dispersed in solution.

\section{Experimental section}

\section{Materials and instruments}

SWCNTs were purchased from Acros (1-2 nm in diameter with lengths of $5-30 \mu \mathrm{m}$ ) and used as received. The synthesis and structure characterization of PEG-diPBI can be found elsewhere. ${ }^{27}$ Other reagents were purchased from Sinopharm Chemical Reagent Co, Ltd. and used without further purification, unless otherwise specified. UV-vis absorption spectra were recorded on a TV-1901 spectrophotometer. Raman spectra were measured in 
solution by RFS100/S Raman Spectrometer $\left(\lambda_{\text {exc }}=1064 \mathrm{~nm}\right)$. For transmission electron microscopy (TEM) measurements, a JEOL JEM-2200FS TEM operating at $200 \mathrm{kV}$ was used. The surface microstructure was examined by SEM (6700F) instrument.

\section{Preparation of PEG-diPBI modified SWCNT}

Typically, commercially available SWCNTs and PEG-diPBI were mixed in $\mathrm{pH}=10$ buffered solution to yield a PEG-diPBI/ SWCNT complex at ratio of $1: 1$ by weight. The resulting mixture was sonicated for $10 \mathrm{~min}$, followed by centrifugation (5000 rpm, $5 \mathrm{~min}$ ) to remove nondispersed SWCNTs.

\section{Antimicrobial test with SWCNT solution}

$300 \mu \mathrm{l}$ PEG-diPBI modified SWCNT solution $\left(0.5 \mathrm{mg} \mathrm{m}^{-1}\right)$ and $900 \mu \mathrm{l}$ the bacterial cell suspensions $\left(1 \times 10^{5} \mathrm{cfu} \mathrm{ml}^{-1}\right)$ were introduced into micro centrifuge tubes. The tubes were kept rotating on a shaker for designed treatment time at $37^{\circ} \mathrm{C}$.

\section{Results and discussion}

\section{Preparation of PEG-diPBI modified SWCNT}

In previous investigation, we applied lysine and PEG modified tetrachloroethylene as protective agents to disperse SWCNTs. ${ }^{26}$ In order to increase the $\pi-\pi$ interactions between aromatic core and CNTs, we attempt to use diperylene bisimide, which has larger aromatic cores than PBI. ${ }^{27}$ The chemical structure of PEGdiPBI was illustrated in Scheme 1. The PEG-diPBI molecule $(2.2 \mathrm{~nm}$ in width) contains a polycyclic aromatic core $(1.3 \mathrm{~nm}$ in length) aiming for $\pi-\pi$ interaction with SWCNT, while the end hydrophilic PEG segments with molecular weight of 2000 Da are designed to confer water solubility and protect dispersed SWCNTs from agglomeration. Compared to a classic amphiphiles or surfactants, polycyclic aromatic core provides strong $\pi-\pi$ interactions in addition to hydrophobic effects.

It should be noted that when the weight ratio of PEG-diPBI and SWCNT is $1: 1$, the dispersion time is moderate (10 $\mathrm{min})$. However, if the ratio of PEG-diPBI and SWCNT is lower than $1: 1$, it was found that the time needed for dispersion is much longer (exceed 1 hour). When the weight ratio exceeds $1: 1$, more PEG-diPBI is needed but no more SWCNTs could be dispersed, which results in the difficulty to remove excess PEG-
diPBI. Thus, the weight ratio of $1: 1$ was determined to be the best ratio for further experiments.

We mixed PEG-diPBI and SWCNT at a weight ratio of $1: 1$, then sonicated and followed by centrifugation. Supernatant was then separated and characterized. The successful absorption of PEG-diPBI on SWCNT is firstly supported from visual observation (Fig. 1a).

Pristine SWCNT is phase separated from water without dispersant, while the PEG-diPBI water solution at $\mathrm{pH}=10$ is green. In contrast, their mixture displays black, indicating absorption of PEG-diPBI onto SWCNT. The SWCNT dispersions after the sonication were further characterized using UV-vis spectroscopy. As shown in Fig. 1b, the peaks range from 600 to $700 \mathrm{~nm}$ could not be found due to the low concentration of SWCNT $\left(0.1 \mathrm{mg} \mathrm{ml}^{-1}\right)$. Thus, the concentration of SWCNT was increased to $0.19 \mathrm{mg} \mathrm{ml}^{-1}$. As shown in Fig. 1c, peaks at 628 and $693 \mathrm{~nm}$ were observed, which exhibited the characters of the PEG-diPBI. ${ }^{27}$ Different from the spectra of pure PEG-diPBI, the mixture of PEG-diPBI and SWCNT display two peaks in this region, i.e., 653 and $720 \mathrm{~nm}$ which correlate to the von Hove singularities, ${ }^{28}$ indicating the homogeneous dispersion of SWCNTs in aqueous solution.

The dispersion of SWCNTs was further demonstrated by an increase in the Raman spectra $I_{\mathrm{D}} / I_{\mathrm{G}}$ ratio. As shown in Raman spectra (Fig. 2a), the intensity of the D band was reduced after dispersion, suggesting that some of the structural imperfections and impurities in SWCNTs might be annealed during the dispersion. For the mixture of PEG-diPBI/SWCNT, the $I_{\mathrm{D}} / I_{\mathrm{G}}$ ratio of SWCNT is 0.4 before adding PEG-diPBI, but decreases to 0.24 upon absorbing PEG-diPBI. The obvious decrease of the $I_{\mathrm{D}} / I_{\mathrm{G}}$ value and RBM region of Raman spectra demonstrates that the SWCNT bundles are effectively exfoliated by PEG-diPBI. ${ }^{29}$ Compared to the curve of bundled SWCNT $(102,119,139,166$, $182,214,238,257,272,292,322$, and $339 \mathrm{~nm}$ ), only peaks at 180, 263, 282, 320, and $336 \mathrm{~nm}$ were observed (Fig. 2b). The decrease of the peak number in PEG-diPBI modified SWCNT confirmed the dispersion of SWCNTs.

Finally, the effective dispersion of SWCNTs by PEG-diPBI was directly visualized using electron microscopy technique. Before adding PEG-diPBI, SWCNTs existed as aggregated bundles. Upon mixing with PEG-diPBI aqueous solution, SWCNTs were observed as isolated tubes with length of approximately

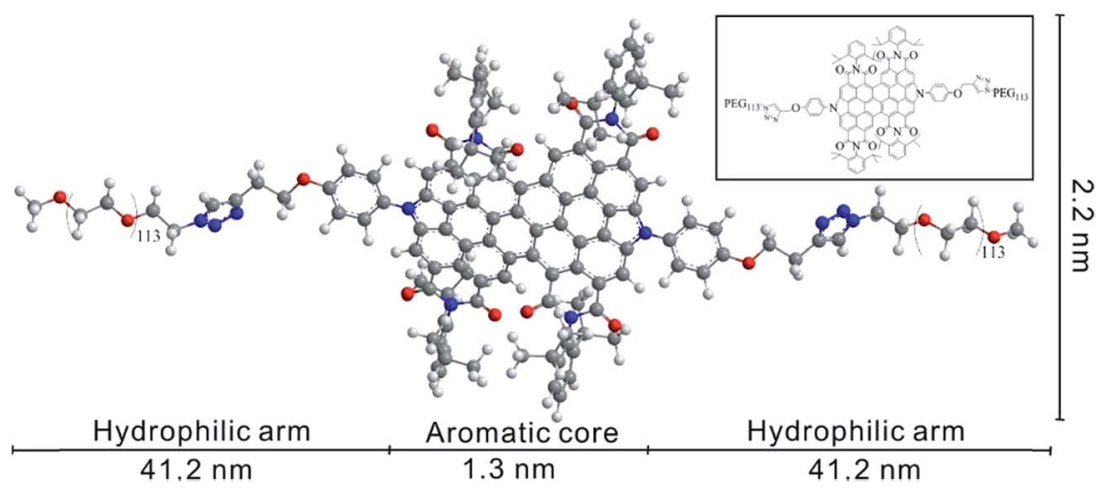

Scheme 1 The extended structure of PEG-diPBI. Inset: the corresponding chemical structure of PEG-diPBI. 

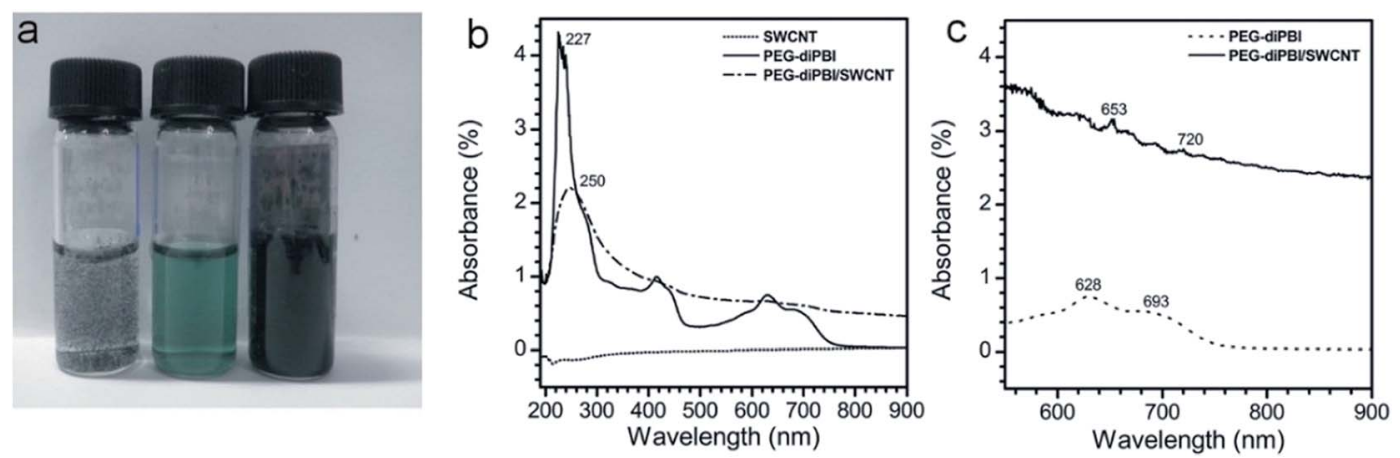

Fig. 1 (a) Photographs of SWCNT at buffer (left), PEG-diPBI in buffer (middle) and 4 PEG-diPBI/SWCNT (right). (b) Absorption spectra of SWCNT, PEG-diPBI and the same dispersion after the addition of SWCNT $\left(0.1 \mathrm{mg} \mathrm{ml}^{-1}\right.$ ). (c) PEG-diPBI in buffered (pH =10) without and with the addition of SWCNT at the concentration of $0.19 \mathrm{mg} \mathrm{ml}^{-1}$.

hundreds of nanometers. As shown in Fig. 3, TEM images of the SWCNT sample revealed debundled nanotubes, while dispersivity observations indicate that the sample is well dispersed in aqueous solutions and hydrophilic due to the presence of PEG functional groups. It should be noted that more than one TEM image (Fig. 3a-c) were provided due to the dilute PEGdiPBI modified SWCNT. It should be mentioned that it is difficult to observe the SWCNT due to the very small diameter and low contrast. ${ }^{26}$ In order to provide more detail of PEI-diPBI/ SWCNT, three additional TEM images were provided in Fig. S1. $\dagger$

The mechanism of the PEG-diPBI modified SWCNT was presented. Initially, SWCNTs in the solution were aggregated due to the hydrophobic interactions; however, after the addition of PEG-diPBI, the aromatic interaction between di-perylene cores and nanotube's sidewall resulted in the adsorption. Meanwhile, long PEG side chains helped to stabilize the SWCNTs in aqueous solution.

\section{Antimicrobial test with SWCNT solution}

Then, to study the bacterial growth inhibition efficiency of the samples, bacterial growth curves were monitored as shown in
Fig. 4. The optical density at $600 \mathrm{~nm}$ (OD600) for the bacteria suspension reveals the bacterial growth. The effect of the treatment time on the antibacterial activity on PEG-diPBI modified SWCNTs is also investigated. For the control, the OD600 values reached 0.405 for $E$. coli after 5 h. It can be seen that unmodified SWCNTs did not cause much delays in bacterial growth, suggesting that only SWCNTs without PEG-diPBI modification did not exhibit significant antibacterial activity to bacterial cells. As for PEG-diPBI modified SWCNTs, the bacterial growth was significantly delayed and suppressed. It is speculated that smaller size and higher mobility can enhance the PEG-diPBI modified SWCNT physical punctures on bacteria, leading to the observed superior antibacterial activity from individually dispersed SWCNT samples. Importantly, no significant growth for the bacteria was observed at PEG-diPBI modified SWCNTs dispersions, demonstrating that the PEGdiPBI/SWCNTs could inhibit bacterial growth effectively. The results also strongly complement our suggestion that individually dispersed SWCNTs have a better antibacterial activity than SWCNT aggregates. The reason is that individual SWCNTs are more mobile in the solution which enhances their physical
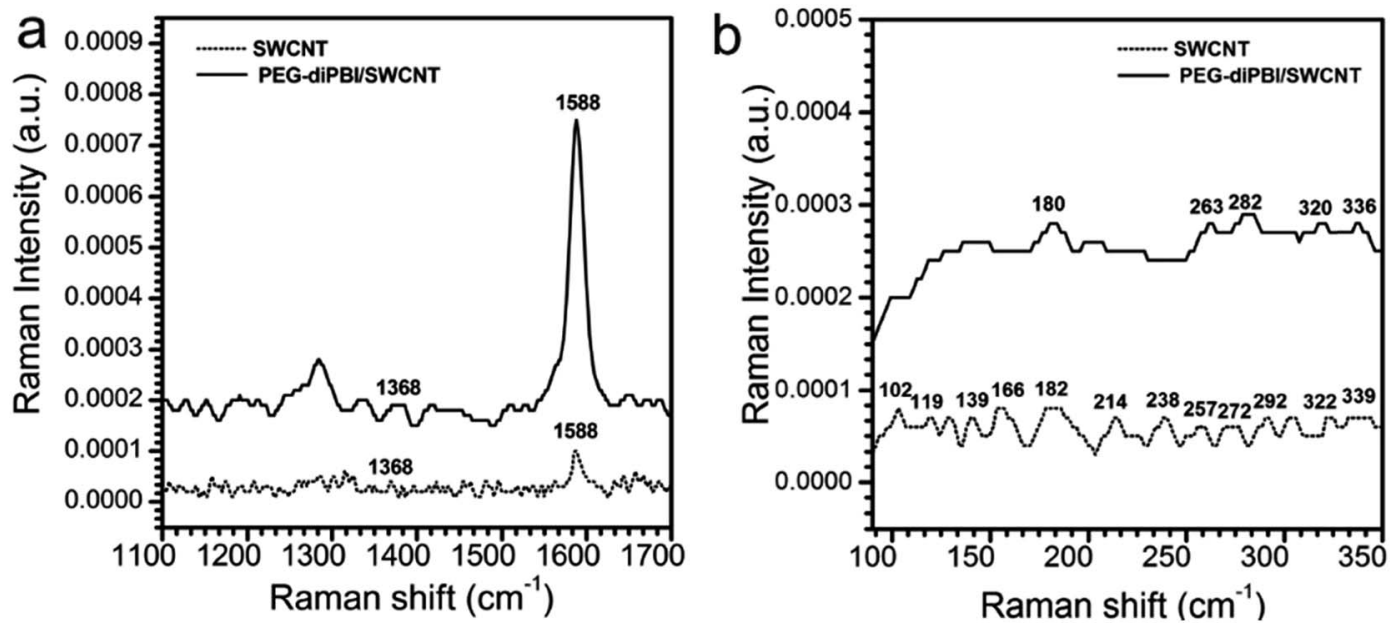

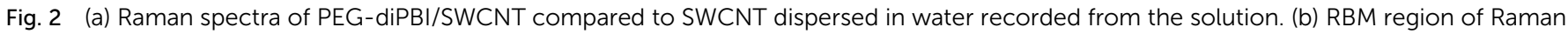
spectra of PEG-diPBI/SWCNT. 


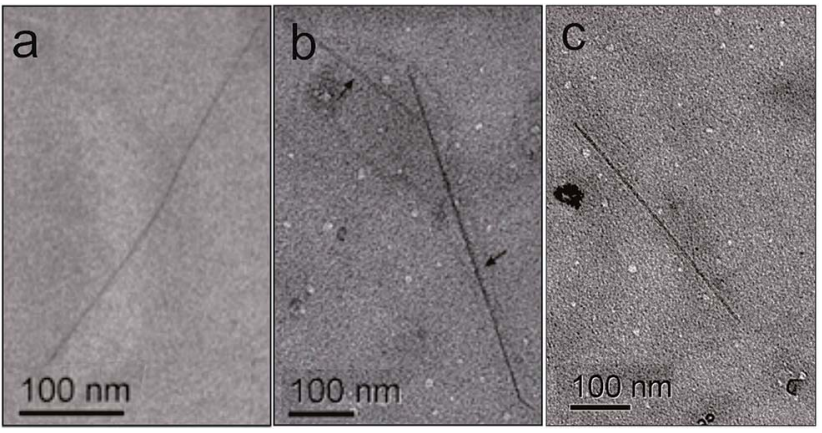

Fig. 3 (a-c) TEM image of SWCNT after the addition of PEG-diPBI $\left([\right.$ PEG-diPBI $]=1 \mathrm{mg} \mathrm{ml}^{-1},[$ SWCNT $\left.]=1 \mathrm{mg} \mathrm{ml}^{-1}\right)$.

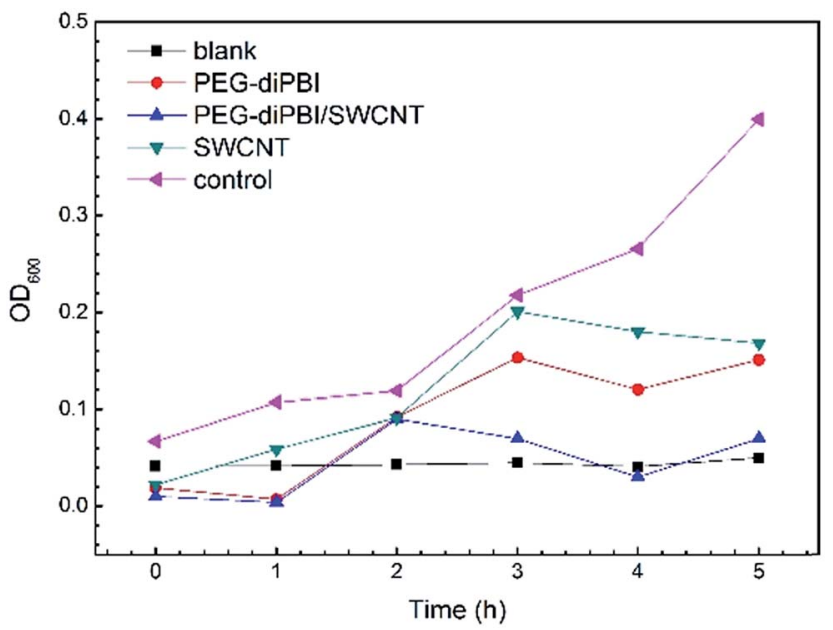

Fig. 4 OD growth curves of $E$. coil in broth at $37^{\circ} \mathrm{C}$ after the bacterial cells had been treated with PEG-diPBI modified SWCNT.

punctures on bacteria. This agrees well with previously reported results on CNTs debundled and dispersed CNTs with high antibacterial activities. As shown in Fig. 4, polyethylene glycol modified diperylene bisimide have antibacterial properties (red plot), but their antibacterial activities is not as good as PEIdiPBI/SWCNT (blue plot).

SEM images of $E$. coli cells exposed to SWCNT showed distinct morphological changes (Fig. 5). In the initial stage, $E$. coli maintain normal cell physiology (Fig. 5a). After the $2 \mathrm{~h}$ incubation with SWCNTs, most of the E. coli cells lost their cellular integrity and became flattened (Fig. 5b), indicating irreversible cell damage and cell death. After $4 \mathrm{~h}$ incubation with SWCNTs, more E. coli cell membrane disrupt and lysis (Fig. 5c). The images in Fig. 5d demonstrated that the cells damaged after the $8 \mathrm{~h}$ incubation, verifying that SWCNTs with high potential damage to cellular membranes. The reason why only few SWCNTs are visualized in our SEM images may be attributed to the individually dispersed SWCNTs that are 1$2 \mathrm{~nm}$ in diameter are difficult to observe in SEM.

To further understand the mechanisms of the antibacterial activity of the diPBI-PEG SWCNTs, this investigation examines how the diPBI-PEG modified SWCNTs interact with bacterial
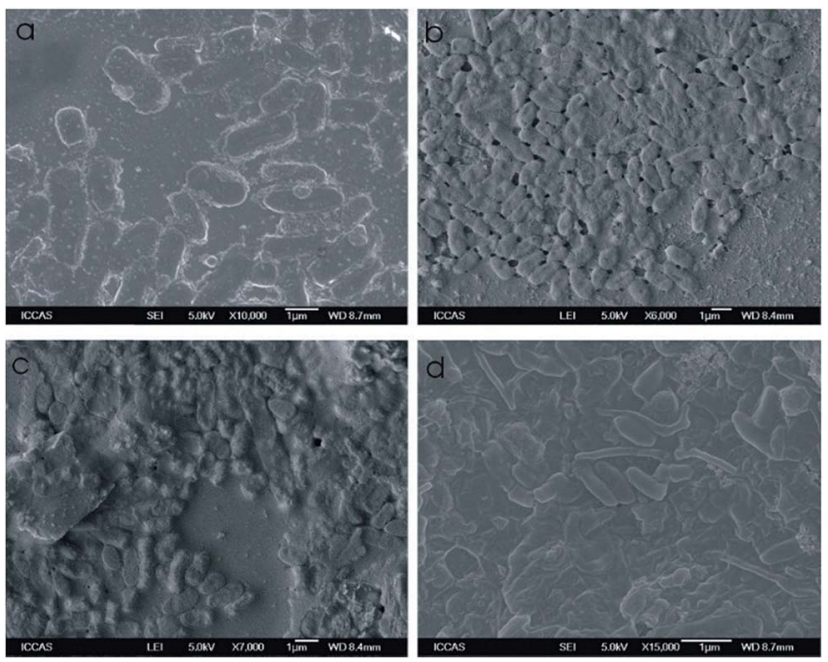

Fig. 5 SEM image of bacterial cells treated with PEG-diPBI modified SWCNTs, (a) 0 h, (b) 2 h, (c) 4 h, (d) 8 h.

cells using fluorescence microscopy (Fig. 6). Fluorescent dyes (DAPI/PI), as an indicator of cell membrane damage, are used to assess the antibacterial activity of all samples. Living and dead cells are observed as blue and red, respectively. Fig. 6a shows a fluorescence microscopy image of the control sample where no diPBI-PEG modified SWCNTs are added. All the cells were alive (blue). Fig. $6 \mathrm{~b}$ shows images of the cells treated with the diPBI-PEG modified SWCNTs at $0.5 \mathrm{mg} \mathrm{ml}^{-1}$. The toxic effects of the surfactants on bacteria can be attributed to a disruption of the cell membrane.

\section{Mechanism of antimicrobial activities}

We suggest the toxicity of dispersed and debundled nanotubes might stem from increased contact opportunities with the cells. The debundled and disaggregated conformation of the SWCNT may increase contact opportunities between the bacteria and the nanotubes, thereby increasing the apparent toxicity evidenced in the membrane integrity. So, effective dispersion of SWCNTs plays an essential role in inactivation of bacterial cells. Furthermore, the introduction of PEG-diPBI not only improve the dispersion of SWCNTs but also increase their antibacterial activities. We hypothesize that the chance of bacteria being
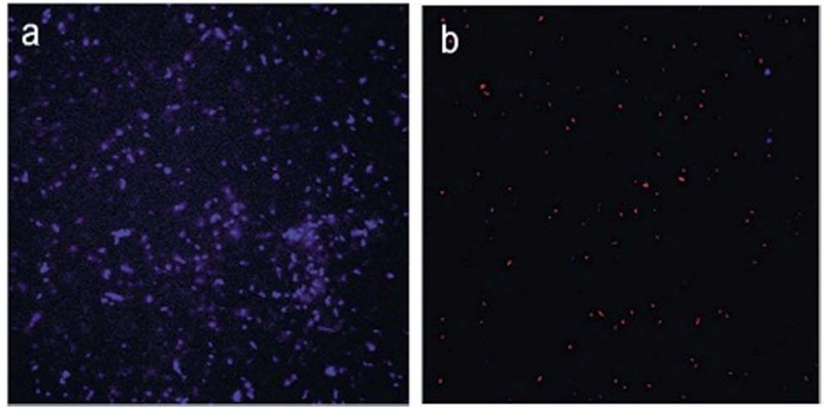

Fig. 6 Fluorescence images of bacterial cells treated without (a) and with (b) PEG-diPBI modified SWCNTs stained by DAPI/PI dyes. 


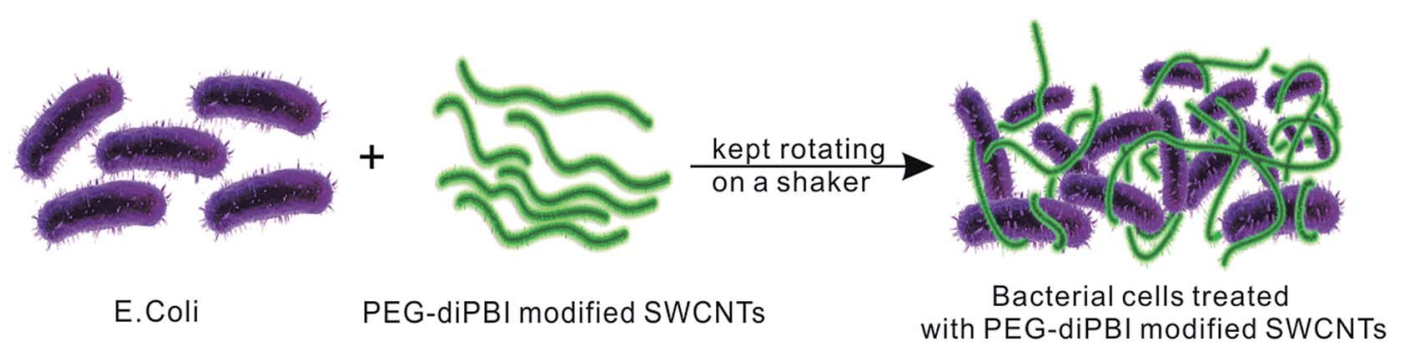

Fig. 7 Mechanism for the interaction between E. coli and PEG-diPBI modified SWCNTs.

punctured by SWCNTs is higher under the longer incubation time, which results in higher cell death rates. The antibacterial mechanisms of SWCNT to bacterial cells were due to the membrane damage by direct contact with SWCNTs (Fig. 7).

\section{Conclusions}

In summary, we have succeeded in dispersing SWCNTs in water using PEG-diPBI as an efficient stabilizing agent. The combination of $\pi-\pi$ stacking, van der Waals forces, and hydrophobic interactions could generate the efficient dispersion of SWCNTs. Besides, the antibacterial activity of PEG-diPBI modified SWCNT was demonstrated, which paves a new way for dispersing and manipulating SWCNTs and might have potentials in sensing, drug delivery, and nanodevices.

\section{Acknowledgements}

We grateful appreciated financial support from National Natural Science Foundation of China (21434008, 51225306).

\section{References}

1 B. Liu, F. Wu, H. Gui, M. Zheng and C. Zhou, ACS Nano, 2017, 11, 31-53.

2 R. Alshehri, A. M. Ilyas, A. Hasan, A. Arnaout, F. Ahmed and A. Memic, J. Med. Chem., 2016, 59, 8149-8167.

3 C. Cao, J. B. Andrews, A. Kumar and A. D. Franklin, ACS Nano, 2016, 10, 5221-5229.

4 M. Mollahosseini, E. Karunaratne, G. N. Gibson, J. A. Gascón and F. Papadimitrakopoulos, J. Am. Chem. Soc., 2016, 138, 5904-5915.

5 M. Scardamaglia, C. Struzzi, F. J. Aparicio Rebollo, P. De Marco, P. R. Mudimela, J.-F. Colomer, M. Amati, L. Gregoratti, L. Petaccia, R. Snyders and C. Bittencourt, Carbon, 2015, 83, 118-127.

6 P. Verma, P. Saini, R. S. Malik and V. Choudhary, Carbon, 2015, 89, 308-317.

7 G. Chen, R. C. Davis, D. N. Futaba, S. Sakurai, K. Kobashi, M. Yumura and K. Hata, Nanoscale, 2016, 8, 162-171.

8 D. Tasis, N. Tagmatarchis, A. Bianco and M. Prato, Chem. Rev., 2006, 106, 1105-1136.

9 D. A. Britz and A. N. Khlobystov, Chem. Soc. Rev., 2006, 35, 637-659.
10 C. Backes, C. D. Schmidt, K. Rosenlehner, F. Hauke, J. N. Coleman and A. Hirsch, Adv. Mater., 2010, 22, 788-802.

11 M. Tange, T. Okazaki, Z. Liu, K. Suenaga and S. Iijima, Nanoscale, 2016, 8, 7834-7839.

12 J.-H. Lee, S.-M. Yoon, K. K. Kim, I.-S. Cha, Y. J. Park, J.-Y. Choi, Y. H. Lee and U. Paik, J. Phys. Chem. C, 2008, 112, 15267-15273.

13 C. Backes, F. Hauke and A. Hirsch, Adv. Mater., 2011, 23, 2588-2601.

14 F. Ernst, T. Heek, A. Setaro, R. Haag and S. Reich, Adv. Funct. Mater., 2012, 22, 3921-3926.

15 S. Kang, M. Herzberg, D. F. Rodrigues and M. Elimelech, Langmuir, 2008, 24, 6409-6413.

16 S. Kang, M. S. Mauter and M. Elimelech, Environ. Sci. Technol., 2008, 42, 7528-7534.

17 S. Kang, M. S. Mauter and M. Elimelech, Environ. Sci. Technol., 2009, 43, 2648-2653.

18 S. Liu, L. Wei, L. Hao, N. Fang, M. W. Chang, R. Xu, Y. Yang and Y. Chen, ACS Nano, 2009, 3, 3891-3902.

19 P. G. Luo, H. Wang, L. Gu, F. Lu, Y. Lin, K. A. Christensen, S.-T. Yang and Y.-P. Sun, ACS Nano, 2009, 3, 3909-3916.

20 W. Yuan, G. Jiang, J. Che, X. Qi, R. Xu, M. W. Chang, Y. Chen, S. Y. Lim, J. Dai and M. B. Chan-Park, J. Phys. Chem. C, 2008, 112, 18754-18759.

21 C. Nie, C. Cheng, L. Ma, J. Deng and C. Zhao, Langmuir, 2016, 32, 5955-5965.

22 S. Pramanik, J. Hazarika, A. Kumar, L. Aidew, A. K. Buragohain and N. Karak, ACS Sustainable Chem. Eng., 2014, 2, 2510-2518.

23 C.-F. de Lannoy, D. Jassby, K. Gloe, A. D. Gordon and M. R. Wiesner, Environ. Sci. Technol., 2013, 47, 2760-2768.

24 Y. J. Kang, S.-J. Chun, S.-S. Lee, B.-Y. Kim, J. H. Kim, H. Chung, S.-Y. Lee and W. Kim, ACS Nano, 2012, 6, 6400-6406.

25 C. D. Vecitis, K. R. Zodrow, S. Kang and M. Elimelech, ACS Nano, 2010, 4, 5471-5479.

26 Y. Sun, W. Fu, Z. Li and Z. Wang, Langmuir, 2014, 30, 86158620.

27 Y. Cui, Y. Wu, Y. Liu, G. Yang, L. Liu, H. Fu, Z. Li, S. Wang, Z. Wang and Y. Chen, Dyes Pigm., 2013, 97, 129-133.

28 V. Zorbas, A. Ortiz-Acevedo, A. B. Dalton, M. M. Yoshida, G. R. Dieckmann, R. K. Draper, R. H. Baughman, M. JoseYacaman and I. H. Musselman, J. Am. Chem. Soc., 2004, 126, 7222-7227.

29 T. Ogoshi, Y. Takashima, H. Yamaguchi and A. Harada, J. Am. Chem. Soc., 2007, 129, 4878-4879. 